\title{
QUALIDADE DE VIDA E BEM-ESTAR ESPIRITUAL EM PESSOAS VIVENDO COM HIV/AIDS ${ }^{1}$
}

\author{
Prisla Ücker Calvetti \\ Marisa Campio Muller ${ }^{\#}$ \\ Maria Lúcia Tiellet Nunes
}

\begin{abstract}
RESUMO. Este estudo teve o objetivo de avaliar a qualidade de vida e bem-estar espiritual em pessoas vivendo com HIV/Aids. A amostra foi constituída de 200 pessoas vivendo com HIV/Aids (111 mulheres e 89 homens), de 19 a 67 anos, 143 sintomáticos e 57 assintomáticos. Do total, 62,5\% faziam uso de terapia anti-retroviral. Os resultados apontam para a correlação significativa entre os domínios da qualidade de vida (WHOQOL-HIV bref) e bem-estar espiritual (SWBS). O domínio da qualidade de vida, espiritualidade, religiosidade e crenças pessoais e a subescala bem-estar religioso apresentaram os escores mais altos. Obtiveram-se correlações altamente significativas entre os domínios da qualidade de vida física, relações sociais e espiritualidade, religiosidade e crenças pessoais, e os do bem-estar religioso, bem-estar existencial e bemestar espiritual no grupo sintomático/Aids. Discute-se a importância das variáveis religiosidade e espiritualidade no processo de resiliência e de proteção à saúde. A importância da inserção dos fatores de proteção à saúde necessita ser destacada nas pesquisas, nas intervenções do psicólogo e no planejamento de políticas públicas de saúde.
\end{abstract}

Palavras-chave: qualidade de vida, bem-estar espiritual, HIV/Aids.

\section{QUALITY OF LIFE AND SPIRITUAL WELL BEING IN INDIVIDUALS WITH HIV/AIDS}

\begin{abstract}
Current study evaluates the quality of life and spiritual well-being in HIV/Aids people. Two hundred HIV individuals were taken as samples (111 women and 89 men), aged between 19 to 67 years old, 143 symptomatic/Aids and 57 asymptomatic, $62.5 \%$ had taken anti-retroviral therapy. Results point to a significant correlation between the quality of life (WHOQOL-HIV bref) and spiritual well being (SWB). The spiritual, religiosity and personal beliefs and the subscale religious well being had the highest scores. Significant correlations were obtained in symptomatic/Aids group between quality of life concerning physical, social relationship and spiritual, religiosity and personal beliefs with the religious well-being, the existential well being and the spiritual well-being. The importance of the variables religiosity and spirituality within the resilience and health protection process was also discussed. Discussing health protection in research, in psychologist's interventions and in health public policies should be enhanced.
\end{abstract}

Key words: Quality of life, spiritual well-being, HIV/Aids.

\section{CUALIDAD DE VIDA Y BIENESTAR ESPIRITUAL EN PERSONAS QUE VIVEN CON VIH/SIDA}

RESUMEN. Este estudio tuvo el objetivo de evaluar la cualidad de vida y bienestar espiritual de personas viviendo con VIH/SIDA. La muestra fue de 200 pacientes viviendo con VIH/SIDA (111 mujeres y 89 hombres), de 19 a 67 años, 143 sintomáticos y 57 asintomáticos, del total, 62,5\% hacían uso de terapia antirretroviral. Los resultados apuntan hacia la correlación significativa entre los dominios de la cualidad de vida (WHOQOL-HIV bref) y bienestar espiritual (SWBS). El dominio de la cualidad de vida, espiritualidad, religiosidad y creencias personales como la subescala bienestar religioso presentaron los escores más altos. Se obtuvieron correlaciones altamente significativas entre los dominios de cualidad de vida físico, relaciones sociales y espiritualidad, religiosidad y creencias personales con el bienestar religioso, existencial y

1 Apoio: $\mathrm{CNPq}$.

* Psicóloga. Mestre em Psicologia Clínica. Doutoranda em Psicologia pela Pontifícia Universidade Católica do Rio Grande do Sul-PUCRS.

\# Psicóloga. Doutora em Psicologia. Professora Titular da Faculdade de Psicologia da PUCRS.

II Doutora em Psicologia. Professora Coordenadora do Programa de Pós-graduação em Psicologia da Pontifícia Universidade Católica do Rio Grande do Sul-PUCRS. 
espiritual del grupo sintomático/SIDA. Se discute la importancia de las variables religiosidad y espiritualidad en el proceso de resiliencia y de protección a la salud. La importancia de la inserción de los factores de protección a la salud necesita ser destacada en las investigaciones, en las intervenciones del psicólogo y en el planeamiento de políticas públicas de salud.

Palabras-clave: Cualidad de vida, bienestar espiritual, VIH/Sida.

No mundo existem milhões de pessoas infectadas pelo Vírus da Imunodeficiência Adquirida (HIV), sendo necessários investimentos em ações educativas e de prevenção, além do tratamento da saúde para a melhoria da qualidade de vida dos portadores do HIV, em relação não apenas ao bem-estar físico, mas também aos aspectos psicossociais, incluindo a religiosidade e a espiritualidade (WHOQOL GROUP, 2003). As pesquisas sobre espiritualidade em relação aos portadores de HIV/Aids são escassas no Brasil, mas elas já existem em maior número no âmbito internacional.

A Síndrome da Imunodeficiência Adquirida (Aids) tem sido um dos grandes problemas de saúde pública no Brasil, sendo crescente a incidência da doença. De 1980 a 2005 foram notificados 371.827 casos de Aids, registrados com contagem de linfócitos TCD4+ menor que $350 \mathrm{~mm}^{3}$; sendo, de julho de 2004 a junho de 2005, notificados 19.000 casos novos. Evidencia-se o crescimento da epidemia entre as populações mais vulneráveis sociodemograficamente (Boletim Epidemiológico Aids e DST, 2005).

Desde o advento dos anti-retrovirais, tem sido também foco da atenção dos programas de intervenção a adesão ao tratamento, pois o uso de medicação tem prolongado o tempo de vida e auxiliado na qualidade de vida (Remor, 2002a). Nesse âmbito, torna-se importante a intervenção dos psicólogos para a efetividade do tratamento no atendimento às pessoas com HIV/Aids (Brannon \& Feist, 2001; Remor, 2002b).

Existem evidências sobre a relação do sistema imunológico e do sistema nervoso com fatores psicossociais quanto à infecção do HIV. Seidl, Zannon e Trócoli (2005) destacam que aspectos relacionados a hábitos e estilos de vida, estresse e estratégias de coping e/ou enfrentamento e apoio social estão implicados no processo saúde-doença.

No contexto de saúde, têm sido realizadas pesquisas e intervenções em relação ao tema da qualidade de vida entendida como a harmonização de diferentes modos de viver e dos domínios (níveis/aspectos) físico, mental, social, cultural, ambiental e espiritual (Fleck, Borges, Bolognesi \& Rocha, 2003). Neste âmbito, fundamenta-se a necessidade de avaliar a relação entre bem-estar espiritual e qualidade de vida em pessoas que vivem com HIV/Aids.
Torna-se fundamental diferenciar e esclarecer o entendimento acerca de religiosidade, espiritualidade e crenças pessoais. A espiritualidade se refere às questões de significado da vida e da razão de viver, independentemente de crenças e práticas religiosas. Por religiosidade entende-se a medida em que um indivíduo acredita, segue e pratica uma religião Crenças pessoais são valores que a pessoa sustenta e que formam a base de seu estilo de vida e de comportamento (Fleck, Borges, Bolognesi \& Rocha, 2003; Paiva, 2004).

Neste âmbito, na literatura científica também se destaca o construto de bem-estar espiritual, que se refere à percepção subjetiva da pessoa em relação à sua crença. Este construto se constitui de dois diferentes componentes, um religioso e um existencial - o de sentido de propósito e satisfação de vida. Tais aspectos são avaliados através da escala de bem-estar espiritual (SWBS), desenvolvida por Paloutizian e Ellison (1982) e validada para o Brasil por Volcan, Sousa, Mari \& Horta (2003).

A espiritualidade possui relação estreita com a melhora da qualidade de vida de pacientes com doenças crônicas (Volcan, Sousa, Mari \& Horta, 2003). Estudo realizado com pacientes com HIV/Aids revelou que aqueles que apresentavam escores mais elevados de bem-estar espiritual tendiam a ser mais esperançosos (Fleck, Borges, Bolognesi \& Rocha, 2003). Outros estudos também apontam que a religiosidade e espiritualidade em pessoas com HIV/Aids podem auxiliar como apoio social no ajustamento psicológico (Siegel \& Schrimshaw, 2002) e no enfrentamento da doença (Tangenberg, 2001; Prado et al., 2004), bem como promover a esperança (Newshan, 1998).

Sodergren, Hyland, Crawford e Partridge (2004) destacam que pessoas com crença espiritual tendem a demonstrar positividade na adversidade, como enfrentamento da doença e tratamento da saúde. Hill e Pargament (2003) apontam para o avanço na construção de instrumentos de medida sobre religiosidade $\mathrm{e}$ espiritualidade. Entre os estudos importantes relacionados ao processo saúde-doença estão os aspectos de coping para o processo de enfrentamento da doença (Antoniazzi, Dell'Aglio \& Bandeira, 1998; Lazarus \& Folkman, 1980); e especificamente relacionados a coping espiritualreligioso (CRE) estão os estudos de Pargament, Koening 
e Perez (2000) e de Panzini e Bandeira (2005), referindo que a fé pode ser um modo de lidar com o estresse, estando associada a melhores índices de qualidade de vida. Estudo realizado por Seidl (2005) sobre modalidades de enfrentamento em pessoas que vivem com HIV/Aids ressalta a importância da religiosidade no processo saúde-doença.

Neste âmbito, a Psicologia da Saúde (Matarazzo, 1980) e a Psicologia Positiva (Seligman \& Csikszentmihalyi, 2000) enfatizam a importância da atenção dos psicólogos para o desenvolvimento humano que não enfoque apenas variáveis negativas nas pesquisas e intervenções em Psicologia. Como variáveis positivas relacionadas aos aspectos sadios do desenvolvimento humano destacam esperança, otimismo, resiliência e espiritualidade.

Para Rutter (1990), com destaque também para Pesce, Assis, Santos e Oliveira (2004), a resiliência refere-se à capacidade do indivíduo de relativizar as situações de adversidade em função dos aspectos intrapsíquicos e do ambiente, levando-o a ressignificar a situação-problema. De acordo com Yunes (2003, 2006), faz-se necessário que os estudos sobre a resiliência busquem uma visão sistêmica Diante do exposto, esta pesquisa teve como objetivos avaliar a qualidade de vida e bem-estar espiritual em pessoas que vivem com HIV/Aids, comparar os grupos assintomático e sintomático/Aids e verificar se existe relação entre qualidade de vida e bem-estar espiritual na população em estudo.

\section{MÉTODO}

O método utilizado nesta pesquisa é o de natureza quantitativa, caracterizando-se como um estudo transversal de caráter descritivo, correlacional e de diferença entre grupos.

\section{Caracterização dos participantes}

A amostra da pesquisa foi composta por 200 pessoas com HIV/Aids, do sexo masculino (44,5\%) e do feminino $(55,5 \%)$, de 19 anos a 67 anos de idade $(M=38,5$ $D P=10,12$ ), atendidas em dois serviços de saúde da rede pública de referência para tratamento em HIV/Aids de Porto Alegre - RS. Quanto à escolaridade, a distribuição da amostra indicou que 59,5\% tinham até o ensino fundamental, $32,5 \%$ cursaram o ensino médio completo e $8 \%$ tinham o curso superior completo. Em relação ao estado civil, $41,5 \%$ eram casados ou viviam com o(a) companheiro(a) e 58,5\% não viviam com o(a) companheiro (a). Entre estes últimos, $36 \%$ do total da amostra eram solteiros, $13 / \%$ eram separados ou divorciados e $9,5 \%$ eram viúvos. Cento e quarenta pessoas (70\% da amostra) indicaram ter filhos. Quanto à situação empregatícia, 70,5\% dos participantes referiram ter uma ocupação remunerada. Dos participantes, 93\% referiram ter alguma religião, e destes, 43,5\% eram da religião católica.

O tempo de diagnóstico variou de 4 meses a 21 anos $(M=5,9 ; D P=6,0)$. Os valores da contagem dos linfócitos TCD4+ (células de defesa do organismo) variaram de 6 a 1498 , tendo a média de $460(D P=275)$. Das 169 pessoas $(84,5 \%)$ que tinham no prontuário registro sobre os exames realizados dos últimos 4 meses de tratamento, $51 \%$ estavam com o TCD4+ acima de 350 na amostra estudada. A carga viral (CV) plasmática (medida pela quantidade de cópias virais por mililitro (ml) de sangue periférico variou de indetectável até 365315 cópias $/ \mathrm{ml}$ $(M=21321 D P=54525)$; das 165 pessoas, 82,5\% apresentavam registro de exames dos últimos 4 meses de exames, 33\% indicaram CV indetectável e 49,5\% acima de 80 cópias/ml. Quanto à medicação, 62,5\% das pessoas faziam uso de anti-retroviral (ARV), e dos 143 (71,5\%) participantes sintomáticos/Aids, $87 \%$ tomavam medicação. Destaca-se que $81 \%$ das pessoas não se consideravam doentes e $57 \%$ delas consideraram boa a sua saúde. Quanto à situação médico-clínica, 57 pessoas $(28,5 \%)$ eram assintomáticas e 143 (71,5\%) sintomáticas/Aids (Tabela 1 em resultados e discussão).

\section{Instrumentos}

$\mathrm{Na}$ obtenção dos dados para a realização desta pesquisa foram usados os instrumentos exposto $\mathrm{e}$ descritos a seguir:

- Questionário de dados sociodemográficos e da situação clínica: esse instrumento contém idade, sexo, escolaridade, número de filhos, situação conjugal, situação empregatícia, tempo de diagnóstico, presença de sintomas e/ou doença oportunista (subdivididos em dois grupos: assintomático e sintomático/Aids), uso de terapia anti-retroviral (ARV), células T CD4+ e carga viral $(\mathrm{CV})$.

- O Instrumento de Avaliação da Qualidade de Vida, da Organização Mundial de Saúde (WHOQOL-HIV Bref), em versão brasileira abreviada do instrumento completo (120 itens), validada por Zimpel e Fleck (2005) avalia a qualidade de vida genérica de pessoas que vivem com HIV/Aids e é composto por 31 itens, distribuídos em de 6 domínios (níveis/aspectos): físico (dor e desconforto, energia e fadiga, sono e repouso), psicológico (sentimentos positivos, pensamento, memória, aprendizado e concentração), nível de independência (mobilidade, atividades cotidianas, 
526

dependência de medicação e tratamento, capacidade para o trabalho), relações sociais (relacionamentos, suporte social, atividade sexual), meio ambiente (segurança física, recursos financeiros, cuidados sociais e de saúde, oportunidades de acesso a novas informações e de lazer, ambiente físico e transporte) e espiritualidade/religiosidade/crenças pessoais (crenças pessoais, religiosas e espirituais). As questões são individualmente pontuadas em uma escala tipo likert de 5 pontos, em que 1 indica percepções baixas e negativas e 5 , percepções altas e positivas (WHOQOL GROUP, 2003).

- A Escala de Bem-estar Espiritual (SWBS), versão brasileira validada por Volcan, Sousa, Mari e Horta, (2003) como referência para medida da religiosidade e espiritualidade, era composta por 20 questões, subdivididas em duas subescalas de 10 itens cada, uma de bem-estar religioso e uma de bem-estar existencial. As questões sobre bem-estar religioso contêm referência a Deus, e os itens de bem-estar existencial referem-se à sensação de encontro com o sentido e o comprometimento com algo significativo na vida. As questões são respondidas em escala, de concordo fortemente a discordo fortemente. Os escores da escala são classificados com os respectivos valores para o total: 20 a 40 (baixo), 41 a 99 (moderado) e 100 a 120 (alto), e para as subescalas: 10 a 20 (baixo), 21 a 49 (moderado) e 50 a 60 (alto) (Ellison, 1983; Paloutizian \& Ellison, 1982; Volcan, Sousa, Mari \& Horta, 2003).

A presente pesquisa foi aprovada pela Comissão Científica da Faculdade de Psicologia da PUCRS e pelos Comitês de Ética do Hospital São Lucas da PUCRS (oficio n. 009.2006). Os participantes consentiram na realização da pesquisa e assinaram o Termo de Consentimento Livre e Esclarecido (TCLE).

\section{Procedimentos de coleta de dados}

Os participantes foram convidados em dias de consulta médica, enquanto estavam em sala de espera ou logo após o atendimento. A resposta aos instrumentos, após a assinatura do Termo de Consentimento Livre e Esclarecido, ocorreu no mesmo dia do atendimento médico no serviço de saúde, a que o paciente estava vinculado. A aplicação foi individual, realizada pelo próprio pesquisador em uma sala reservada e em um único encontro de aproximadamente quarenta e cinco minutos.

\section{Análise dos dados}

Os dados foram submetidos à análise de correlação de Spearman, de modo a permitir o estabelecimento de correlações entre bem-estar espiritual e qualidade de vida.
O teste U de Mann-Whitney foi utilizado para verificar a diferença entre os grupos assintomático e sintomático em relação aos dados sociodemográficos e clínicos, à escala de bem-estar espiritual e ao questionário de qualidade de vida. O nível de significância usado é de $p<0.05$, sendo os dados computados no Statistical Package for Social Sciences (SPSS) 11.5.

\section{RESULTADOS}

A seguir, são apresentadas as características sociodemográficas e da situação clínica dos grupos assintomático e sintomático/Aids.

Tabela 1. Caracterização dos Participantes Referente aos Dados Sociodemográficos e da Situação Clínica nos Grupos Assintomáticos (n=57) e Sintomático/Aids (n=143)

\begin{tabular}{|c|c|c|c|c|}
\hline \multirow{2}{*}{ Variável } & \multicolumn{2}{|c|}{ Assintomático } & \multicolumn{2}{|c|}{ Sintomático/Aids } \\
\hline & $N$ & $\%$ & $N$ & $\%$ \\
\hline \multicolumn{5}{|l|}{ Sexo } \\
\hline Masculino & 21 & 36,8 & 68 & 47,6 \\
\hline Feminino & 36 & 63,2 & 75 & 52,4 \\
\hline \multicolumn{5}{|l|}{ Idade } \\
\hline$\leq 29$ anos & 22 & 38,6 & 24 & 16,8 \\
\hline$\geq 30$ anos & 35 & 62,4 & 76 & 84,2 \\
\hline \multicolumn{5}{|l|}{ Escolaridade } \\
\hline$\leq 1^{\circ}$ grau & 32 & 56,1 & 87 & 60,9 \\
\hline$\geq 2^{\circ}$ grau & 25 & 43,9 & 46 & 39,1 \\
\hline \multicolumn{5}{|l|}{ Estado Civil } \\
\hline Vive com companheiro(a) & 28 & 49,2 & 55 & 38,5 \\
\hline Vive sem companheiro(a) & 29 & 50,8 & 88 & 62,5 \\
\hline \multicolumn{5}{|l|}{ Filhos } \\
\hline Sim & 37 & 64,9 & 105 & 73,4 \\
\hline Não & 20 & 35,1 & 38 & 26,6 \\
\hline \multicolumn{5}{|l|}{ Ocupação remunerada } \\
\hline $\operatorname{Sim}$ & 46 & 80,7 & 103 & 72 \\
\hline Não & 11 & 19,3 & 40 & 28 \\
\hline \multicolumn{5}{|l|}{ Religião } \\
\hline Católica & 31 & 54,4 & 66 & 46,2 \\
\hline Outras & 26 & 45,7 & 77 & 53,9 \\
\hline \multicolumn{5}{|l|}{ Como está sua saúde? } \\
\hline Muito Ruim & 0 & 0,0 & 3 & 2,1 \\
\hline Ruim & 1 & 1,8 & 15 & 10,5 \\
\hline Nem Ruim, nem boa & 7 & 12,3 & 37 & 25,9 \\
\hline Boa & 42 & 73,7 & 72 & 50,3 \\
\hline Muito Boa & 7 & 12,3 & 15 & 10,5 \\
\hline \multicolumn{5}{|l|}{$\begin{array}{l}\text { Você se considera doente } \\
\text { atualmente? }\end{array}$} \\
\hline $\operatorname{Sim}$ & 2 & 3,5 & 35 & 24,5 \\
\hline Não & 55 & 96,5 & 108 & 75,5 \\
\hline \multicolumn{5}{|l|}{ Tempo de diagnóstico } \\
\hline$\leq 5$ anos & 39 & 68,4 & 56 & 39,2 \\
\hline$\geq 6$ anos & 18 & 31,6 & 87 & 60,8 \\
\hline \multicolumn{5}{|l|}{ Antiretroviral } \\
\hline Sim & 0 & 0,0 & 125 & 87,4 \\
\hline Não & 57 & 100,0 & 18 & 12,6 \\
\hline
\end{tabular}


O fato de o grupo sintomático/Aids se caracterizar por pacientes que fazem uso de medicação antiretroviral tende a influenciar para uma reorganização da vida. Também esses participantes apresentam maior tempo de diagnóstico, se comparados com o grupo assintomático, bem como idade mais avançada. Isso pode estar relacionado ao tempo prolongado de diagnóstico.

Através do teste $\mathrm{U}$ de Mann-Whitney, a comparação entre os grupos dos assintomáticos e dos sintomáticos/Aids apresentou diferenças relacionadas a variáveis sociodemográficas e da situação clínica da doença. O grupo assintomático apresentou diferença entre a faixa etária ( $\leq 29$ anos e $\geq 30$ anos) do grupo assintomático e o mesmo para ocupação remunerada ( $\operatorname{sim}$ e não) e tempo de diagnóstico $(\leq 29$ anos e $\geq 30$ anos). Os resultados estão relacionados ao fato de esse grupo caracterizar-se por ter maior número de adultos jovens e de pessoas trabalhando e menor tempo de diagnóstico.

O grupo sintomático/Aids apresentou resultados significativos em relação às seguintes questões: "como está a sua saúde?" (Muito ruim, ruim, nem ruim nem boa, boa e muito boa); "você se considera doente?" (sim e não) e também ao uso de antiretroviral (sim e não). Os resultados apresentados estão relacionados ao fato do grupo caracterizar-se por maior tempo de tratamento medicamentoso e então a sua saúde manter-se estabilizada.

Tabelas 2. Médias (M) e Desvios-Padrão (DP) dos Domínios do WHOQOL-HIV Bref nos Grupos Assintomático ( $(n=57)$ e Sintomático/Aids $(n=143)$

\begin{tabular}{lccc}
\hline Variável & Assintomático & Sintomático/Aids & $\boldsymbol{p}$ valor \\
\hline \multicolumn{1}{c}{ Domínios } & & & \\
Físico & $16,94(2,07)$ & $14,55(3,55)$ & $0,00 *$ \\
Psicológico & $14,18(2,04)$ & $13,43(2,90)$ & 0,16 \\
Nível de Independência & $15,57(2,18)$ & $13,44(2,95)$ & $0,00 *$ \\
Relações sociais & $14,54(2,37)$ & $13,89(2,68)$ & 0,10 \\
Meio ambiente & $13,91(1,63)$ & $13,55(1,99)$ & 0,39 \\
Espirit./relig./crenças pessoais & $16,17(2,68)$ & $15,90(3,06)$ & 0,70 \\
\hline \multicolumn{1}{c}{ Subescalas } & & & \\
Bem-estar Religioso & $52,50(8,87)$ & $51,02(10,31)$ & 0,50 \\
Bem-estar Existencial & $45,89(9,74)$ & $43,77(11,34)$ & 0,26 \\
Bem-estar Espiritual & $98,40(16,93)$ & $94,80(19,46)$ & 0,28 \\
\hline
\end{tabular}

* estatisticamente significativo para $\mathrm{p}<0,05$

As médias encontradas nos domínios da qualidade de vida e nas subescalas do bem-estar espiritual nos grupos assintomáticos e sintomáticos/Aids se apresentam semelhantes, conforme a Tabela 2, porém são observáveis escores mais altos no grupo assintomático, sendo o domínio físico o mais elevado, seguido do domínio espiritualidade, religião e crenças pessoais. No grupo sintomático/Aids, o escore mais alto foi o domínio espiritualidade, religião e crenças pessoais, e o mais baixo, o domínio psicológico. Observa-se que o domínio espiritualidade, religião e crenças pessoais se encontra em destaque em ambos os grupos. Este resultado poder ser compreendido como um indício de enfrentamento criativo e positivo. Pode-se pensar que o enfrentamento da doença contribua para o desenvolvimento deste aspecto, bem como o inverso, sendo este um recurso para o processo de resiliência.

Também se pode refletir que o baixo escore no domínio psicológico, no grupo sintomático em relação ao assintomático, esteja relacionado ao fato de a maioria dos pacientes encontrarem-se em terapia antiretroviral. O tratamento medicamentoso, além do diagnóstico, tende a gerar impacto e sentimentos negativos, tais como ansiedade e tristeza, tornando-se necessária uma readaptação na convivência com a doença. Neste estudo, pode-se pensar que o fato de a pessoa soropositiva para o HIV ter apresentado ou ainda estar apresentando sintomas propicia o desenvolvimento da religiosidade-espiritualidade como fonte de apoio emocional e/ou social.

A análise do teste de Mann-Whitney (Tabela 2) indicou que, em relação à qualidade de vida, o grupo sintomático/Aids apresentou diferença em comparação ao assintomático nos domínios físico $(p=0,00)$ e nível de independência $(p=0,00)$, e não se obteve diferença significativa entre os grupos em relação ao bem-estar espiritual.

Tabela 3. Correlação de Spearman $\left(r_{s}\right)$ entre Qualidade de Vida e Bem-Estar Espiritual nos Grupos Assintomático ( $n=57)$ e Sintomático/Aids $(n=143)$

\begin{tabular}{lccc}
\hline \multicolumn{1}{c}{ Domínios } & \multicolumn{3}{c}{ Bem-estar } \\
\cline { 2 - 4 } \multicolumn{1}{c}{ Assintomático } & Religioso & Existencial & Espiritual \\
\hline Físico & 0,05 & 0,24 & 0,14 \\
Psicológico & 0,15 & $0,48^{* *}$ & $0,37 * *$ \\
Independência & $-0,14$ & 0,12 & 0,00 \\
Relações sociais & $-0,03$ & 0,12 & 0,05 \\
Meio ambiente & 0,01 & $0,38^{* *}$ & $0,26 * *$ \\
Espirit./relig./crenças pessoais & 0,23 & $0,46^{* *}$ & $0,40^{* *}$ \\
\hline \multicolumn{1}{c}{ Sintomático/Aids } & & & \\
Físico & $0,24 * *$ & $0,49 * *$ & $0,44 * *$ \\
Psicológico & 0,13 & $0,56^{* *}$ & $0,44 * *$ \\
Independência & 0,16 & $0,47^{* *}$ & $0,40^{* *}$ \\
Relações sociais & $0,17 *$ & $0,41^{* *}$ & $0,37 * *$ \\
Meio ambiente & 0,10 & $0,46^{* *}$ & $0,36^{* *}$ \\
Espirit./relig/crenças pessoais & $0,34 * *$ & $0,58^{* *}$ & $0,53 * *$ \\
\hline
\end{tabular}

* $p<0,05 * * p<0,01$ 
Pode-se observar na Tabela 3 que o grupo sintomático/Aids obteve mais correlações significativas entre os domínios da qualidade de vida e bem-estar espiritual do que o grupo assintomático. Apenas no grupo sintomático se obtiveram correlações significativas nos domínios: físico $(p=0,00)$, relações sociais $(p=0,03)$ e espiritualidade, religião e crenças pessoais $(p=0,00)$ na avaliação da qualidade de vida com o bem-estar espiritual. Apenas o grupo sintomático indicou correlações positivas com o bemestar religioso.

O domínio psicológico da qualidade de vida teve correlação altamente significativa nos grupos assintomático $(p=0,00)$ e sintomático $(p=0,00)$ com o bem-estar existencial. Pôde-se observar que, no grupo sintomático, o domínio nível de independência foi correlacionado significativamente com bem-estar existencial $(p=0,00)$ e com bem-estar espiritual total $(p=0,00)$. Os resultados podem estar relacionados ao fato de o grupo sintomático ter maior idade e também utilizar a terapia anti-retroviral, e então propiciar nos pacientes o despertamento do sentido da vida e auxiliar no estímulo ao acompanhamento médico.

Ainda na Tabela 3, destaca-se também que somente no grupo sintomático se evidenciaram correlações significativas entre o domínio relações sociais e bem-estar religioso $(p=0,03)$, bem-estar existencial $(p=0,00)$ e bem-estar espiritual $(p=0,00)$. Pode-se pensar que esse resultado esteja relacionado ao grupo sintomático, pelo fato de este caracterizar-se pelo uso da terapia anti-retroviral, como também pelo acompanhamento no serviço de saúde, sendo a equipe uma possível fonte de apoio social. Nesse sentido, a crença religiosa apresentou-se como fonte de apoio, auxiliando numa melhor convivência com o diagnóstico do HIV/Aids.

\section{DISCUSSÃO}

As pesquisas relacionadas ao tema HIV/Aids, conforme Thompson (1999) e de Prado et al. (2004), têm apontado a importância do apoio social no enfrentamento da doença. Em estudo realizado com pessoas soropositivas para o HIV, Dyer, Patsdaughter, McGuinness, O`Connor e DeSantis (2004) referem que as que apresentam processos de resiliência têm enfrentado a situação de enfermidade com maior positividade.

As crenças religiosas e espirituais têm demonstrado ser um recurso auxiliar no enfrentamento de eventos estressores, como o processo saúde-doença e o tratamento da saúde na Psicologia da Saúde (Straub, 2005). Neste estudo, o bem-estar religioso pode ser entendido como apoio social, contribuindo para uma sensação de conforto que tende a auxiliar na convivência com o HIV/Aids. Pode-se pensar que, pela enfermidade, as pessoas tendem a desenvolver religiosidade como uma fonte de apoio social

O bem-estar espiritual pode ser destacado como uma das variáveis presentes na capacidade de resiliência e protetor da saúde. Esta pode auxiliar as pessoas que vivem com HIV/Aids na manutenção e diminuição de agravos do processo saúde-doença, contribuindo para o desenvolvimento da qualidade de vida. Pode-se observar que as pessoas soropositivas, em sua maioria, consideram-se não-doentes, com uma boa saúde. A resiliência pode ser desenvolvida também conforme a vivência e o enfrentamento de situações adversas, como, por exemplo, o HIV/Aids, levando a pessoa ao seu fortalecimento.

A Psicologia Positiva ressalta que as novas investigações relacionadas ao desenvolvimento humano necessitam ser referidas ao bem-estar psicológico e à saúde física, salientando o investimento na descoberta de como as emoções positivas podem auxiliar os indivíduos no ciclo da vida. Experiências de afeto positivo podem auxiliar a pessoa no enfrentamento de estresse crônico, como, por exemplo, a situação de doença como o HIV/Aids, sendo que estas emoções servem como recurso para a superação da adversidade. Neste sentido, a resiliência tem sido relacionada a afetos positivos e de proteção à saúde (Fredrickson, 2003).

Os pacientes sintomáticos/Aids, neste estudo, pontuaram mais correlações significativas entre qualidade de vida (domínios físico; relações sociais; espiritualidade, religiosidade e crenças pessoais) com o bem-estar religioso em comparação ao grupo assintomático. Pode-se apenas inferir que este fato esteja relacionado ao de os participantes do grupo sintomático/Aids indicarem maior freqüência de crenças religiosas em comparação com os outros pacientes (assintomáticos).

Faria e Seidl (2005) mostram a importância da religiosidade no enfrentamento do processo saúdedoença quando o paciente já refere estes aspectos como desenvolvidos na sua vida. Além disso, frisam que este aspecto não deve ser visto somente como relevante para o tratamento e cura da doença, mas sim, no mais amplo entendimento referente ao bem-estar da pessoa.

Desta forma, sugere-se a inclusão, nas novas pesquisas, dos fatores de proteção da saúde, como, por exemplo, o tema da religiosidade-espiritualidade. Alguns estudos brasileiros já têm referido a influência 
de tais aspectos na saúde (Panzini \& Bandeira, 2005; Seidl, 2005; Volcan, Sousa, Mari \& Horta, 2003).

\section{CONSIDERAÇÕES FINAIS}

A Psicologia da Saúde considera os aspectos de religiosidade e espiritualidade como possíveis fontes de apoio social no enfrentamento da doença, especialmente em enfermidades crônicas, como o HIV/Aids. As pesquisas relacionadas à Psicologia Positiva complementam, apontando que as emoções positivas, entre estas a fé e a espiritualidade, podem auxiliar na manutenção e desenvolvimento saudável mesmo em processo de saúde-doença. Neste sentido, busca-se preservar os aspectos sadios do desenvolvimento humano. Entretanto, também se destaca a importância do entendimento de que a fé e a espiritualidade não sejam um afastamento para o enfrentamento da doença, mas que estas possam contribuir como fonte de apoio social no tratamento da saúde.

Faz-se necessária uma reflexão acerca da atenção do psicólogo para o fortalecimento dos processos de resiliência da pessoa vivendo com HIV/Aids no enfrentamento da doença. Ressalta-se a importância do investimento dos aspectos que auxiliam na proteção à saúde, como os estudos sobre espiritualidade, resiliência, coping, otimismo e esperança, contribuindo para uma melhora da qualidade de vida. Conviver com o HIV requer uma readaptação e um reajustamento psicológico a essa situação de estresse.

Para o desenvolvimento de pesquisas futuras, sugere-se a inclusão de variáveis psicológicas positivas e negativas nos estudos relativos ao contexto de saúde. Da mesma forma, sugere-se o aprofundamento dos estudos em relação ao tema da espiritualidade, através da metodologia qualitativa, bem como a necessidade de delineamentos quaseexperimentais com controle de grupos clínicos e nãoclínicos. Além disso, destaca-se a importância dos estudos longitudinais para um possível acompanhamento da influência destas e de outras variáveis no percurso do desenvolvimento humano. Sugere-se também a realização de pesquisaintervenção a fim de investigar, por exemplo, a relação entre intervenção psicológica e variáveis positivas, bem como os efeitos no ajustamento psicológico do indivíduo. Outra sugestão é a construção de um modelo de intervenção enfatizando processos de resiliência para o atendimento às pessoas que vivem com HIV/Aids.
Por fim, é possível afirmar que o estudo ora apresentado buscou contribuir, na área da saúde, para a melhoria da qualidade de vida das pessoas que vivem com HIV/Aids. Além disso, pode auxiliar na discussão da formação do psicólogo e de outros profissionais de saúde para a intervenção nos serviços de saúde, aprimorando o conhecimento do processo saúde-doença, incluindo neste entendimento a espiritualidade, religiosidade e crenças pessoais. Esta compreensão pode fazer diferença na prática clínica, como forma de aprimorar a relação profissional e paciente, bem como na avaliação e comparação de resultados sobre diferentes tratamentos de saúde, também como uma possibilidade de implementar políticas de saúde pública.

\section{REFERÊNCIAS}

Antoniazzi, A., Dell'Aglio, D. \& Bandeira, D. (1998). O conceito de coping: uma revisão teórica. Estudos de Psicologia, 3(2), 273-294.

Boletim Epidemiológico Aids e DST (2005). Ministério da Saúde. Secretaria de Vigilância em Saúde. Programa Nacional de DST e Aids. Ano II (1).

Brannon, L. \& Feist, J. (2001). Psicología de la Salud. Madrid: Paraninfo Thomson Learning.

Dyer, J., Patsdaughter, C., McGuinness, T., O`Connor, C. \& DeSantis, J. (2004). Retrospective Resilience: The power of the patient-provider alliance in disenfranchised persons with HIV/AIDS. Journal of Multicultural Nursing \& Health, 10(1), 57-65.

Ellison, G. (1983). Spiritual well-being: Conceptualization and measurement. Journal of Psychology and Theology, 11(4), 330340.

Faria, J. \& Seidl, E. (2005). Religiosidade e enfrentamento em contextos de saude e doença: revisao da literatura. Psicologia Reflexão e Crítica, 18(3), 381-389.

Fleck, M., Borges, Z., Bolognesi, G. \& Rocha, N. S. (2003). Desenvolvimento do WHOQOL, módulo espiritualidade, religiosidade e crenças pessoais. Revista de Saúde Pública, 37(4), 446-455.

Fredrickson, B. (2003). The value of positive emotions. American Scientist, 91, 330-335.

Lazarus, R. \& Folkman, S. (1980). An Analysis of coping in a middle aged community sample. Journal of Health and Social Behavior, 21, 219-239.

Hill, P. \& Pargament, K. (2003). Advances in the conceptualization and measurement of religion and spirituality. Implications for physical and mental health research. American Psychologist, 58(1), 64-74.

Matarazzo, J. (1980). Behavioural health's challenge to academic, scientific and professional psychology. American Psychologist, 37. 
530

Newshan, G. (1998). Transcending the physical: Spiritual aspects of pain in patients with HIV and/or cancer. Journal of Advanced Nursing, 28(6) 1236-1241.

Paiva, G. (2004). Espiritualidade e qualidade de vida: pesquisas em psicologia. Em E. F. B. Teixeira, M. C. Muller \& J. D. T. Silva (Orgs.), Espiritualidade e Qualidade de Vida (pp. 119-130). Porto Alegre: EDIPUCRS.

Paloutizian, R. \& Ellison, C. (1982). Lonelliss, spiritual well-being and the quality of life. In D. Peplau \& D. Perlman (Eds.), Loneliss: A sourcebook of current theory, research and therapy (pp. 224-235). New York: John Wiley and Sons.

Panzini, R. G. \& Bandeira, D. (2005). Escala de coping religiosoespiritual (escala CRE): elaboração e validação de construto. Psicologia em Estudo, 10(3), 507-516.

Pargament, K., Koening, H. \& Perez, L. (2000). The many methods of religious coping: Development and initial validation of RCOPE. Journal of Clinical Psychology, 56(4), 519-543.

Pesce, R., Assis, S., Santos, N. \& Oliveira, R. (2004), Risco e proteção: em busca de um equilíbrio promotor de resiliência. Revista Teoria e Pesquisa, 20(2), 135-143.

Prado, G., Feaster, D., Schwartz, S., Pratt, I., Smith, L. \& Szapocnik, J. (2004). Religious involvement, coping social support and psychological distress in HIV-seropositive. African American mothers. AIDS and Behavior, 8(3), 221-235.

Remor, E. (2002a). Psiconeuroimunologia e infecção por HIV: realidade ou ficção? Psicologia Reflexão e Crítica, 15(1), 113119.

Remor, E. (2002b). Apoyo social y calidad de vida en la infección por el VIH. Atención Primaria, 30(3), 143-149.

Rutter, M. (1990). Psychosocial resilience and protective mechanisms. In J. Rolf, A. Masten, D. Cicchetti, K. Nuechterlein \& Weintraub, S. (Eds.), Risk and protective factors in the development of psychopathology (pp. 181-214). Cambridge: University.

Seidl, E. (2005). Enfrentamento, aspectos clínicos e sociodemográficos de pessoas vivendo com HIV/Aids. Psicologia em Estudo, 10(3), 421-429.

Seidl, E., Zannon, C. M. \& Trócoli, B. T. (2005). Pessoas vivendo com HIV/Aids: enfrentamento, suporte social e qualidade de vida. Psicologia: Reflexão e Crítica, 18(2), 188-195.

Seligman, M. \& Csikszentmihalyi, M. (2000). Positive Psychology: An introduction. American Psychologist, 55(1) 5-14.
Calvetti et al.

Siegel, K. \& Schrimshaw, E. (2002). The perceived benefits of religious and spiritual coping among older adults living with HIV/AIDS. Journal for the Scientific Study of Religion, 41(1), 91-102.

Sodergren, S., Hyland, M., Crawford, A. \& Partridge, M. (2004). Positivity in illness: Self-delusion or existencial growth? British Journal of Health Psychology, 9, 163-174.

SPSS for Windows (2002) (Release 11.5.0). [Programa de computador], standard version. Chicago (IL): AUTOR.

Straub, R. (2005). HIV e AIDS. Em Psicologia da Saúde (pp. 393424). Porto Alegre: Artmed.

Tangenberg, K. (2001). Surving two diseases: Addiction, recovery, and spirituality among mothers living with HIV disease. Families in Society, 82(5), 517-524.

Thompson, E. (1999). Resiliency in families with a member facing AIDS. In The dynamics of resilient families (pp. 135-159). California: Sage.

Volcan, S. M. A., Sousa, P. L., Mari, J. J. \& Horta, B. L. (2003). Relação entre bem-estar espiritual e transtornos psiquiátricos menores: estudo transversal. Revista de Saúde Pública, 37(4), 440-445.

Yunes, M. A. (2003). Psicologia Positiva e resiliência: o foco no indivíduo e na família. Psicologia em Estudo, 8(n. esp.), 75-84.

Yunes, M. A. (2006). Psicologia positiva e resiliencia: foco no individuo e na familia. Em D. D. Dell Aglio, S. H. Koller \& M. A. M. Yunes (Orgs.), Resiliência e Psicologia Positiva: interfaces do risco à proteção (pp. 69-86). São Paulo: Casa do Psicólogo.

WHOQOL GROUP (2003). Initial steps to development the World Health Organization's Quality of life instrument (WHOQOL) module for international assessment in HIV/AIDS. Aids Care, 15(3), 347-357.

Zimpel, R. \& Fleck, M. (2005). Instrumento WHOQOL. Manual do usuário. Saúde Mental pesquisa e evidência. Departamento de Saude Mental e Dependência Química da OMS. Genebra. Recuperado em 20 de Junho de 2006 de http://www.ufrgs.br/psiq/whoqol_hiv_01.pdf

Recebido em 28/09/2006 Aceito em 01/08/2007

Endereço para correspondência : Prisla Ücker Calvetti. Rua Xavier da Cunha, 999, apto. 228, Cristal, CEP 90830-430, Porto Alegre-RS. E-mail: prisla.calvetti@gmail.com 